\section{COVID-19 and tuberculosis in South Africa: A dangerous combination}

To the Editor: There has been much speculation during the past week about the catastrophe that awaits once coronavirus disease 2019 (COVID-19) establishes itself in the poorest communities of South Africa (SA) and, importantly, in informal settlements. Evidence to date suggests that COVID-19 is efficiently passed from infected individuals via large droplets and hard-surface fomites. ${ }^{[1]}$ Given the housing density and shared toilet and tap facilities in informal settlements, interventions such as frequent hand washing, social distancing and self-isolation will be particularly difficult to achieve. In addition, the disproportionate burden of HIV has left many making dire predictions for the consequences to the people and the health system if, or more appropriately when, COVID-19 reaches these communities. ${ }^{[2]}$ In the wake of World TB Day, we would like to remind readers why tuberculosis (TB) is also an important and complex consideration for vulnerable populations in relation to COVID-19.

At the time of writing (24 March 2020), 554 cases of COVID-19 have been detected and reported in SA, largely among people aged 21 - 60 years. ${ }^{[3]}$ No deaths have yet been reported, and the majority of cases are still imported by people who have recently travelled. ${ }^{[4]}$ In the language of the day, the SA epidemic curve has just begun. Early data from China indicate that individuals most at risk for severe responses to COVID-19 are those aged $>60$ years and those with comorbidities such as chronic respiratory disease, hypertension, diabetes and cardiovascular disease. ${ }^{[5,6]}$ To date, COVID-19 has not been studied in low- and middle-income countries with endemic HIV. With an estimated 7.7 million people living with HIV in SA, ${ }^{[7]}$ concerns about the effect of COVID-19 on people with suppressed immune systems have largely dominated the conversation. Yet HIV is only one piece of the equation.

In SA, an estimated 301000 people developed TB in 2019, nearly $60 \%$ of whom were also HIV-infected ${ }^{[8]}$ It is unclear how COVID-19 may manifest itself in this population. Regardless of HIV status, people with undiagnosed pulmonary TB (PTB), those with drug-resistant TB or complex presentations such as disseminated forms, and those who have only just begun PTB treatment may be at increased risk for severe responses if they become infected with COVID-19. As seen with PTB and silicosis, combined pulmonary diseases can multiply the gravity of the situation. ${ }^{[9,10]}$ Moreover, common COVID-19 symptoms (cough, fever, and to a lesser degree, shortness of breath) overlap with PTB, which may make distinguishing between the two problematic for healthcare workers in high-burden communities. Although sputum production, a common feature of PTB, is less common among individuals with COVID-19, ${ }^{[5]}$ those with TB-HIV co-infection may not present with sputum production. ${ }^{[11]}$ It will be important for clinicians to stay vigilant and consider PTB in their differential diagnosis of COVID-19 alongside the possibility of dual infection with COVID-19 and PTB, especially among people living with HIV. It is equally important that patients with confirmed or presumptive PTB symptoms be prioritised for COVID-19 testing.

TB and COVID-19 could result in poorer treatment outcomes, especially if TB treatment is interrupted. ${ }^{[12]}$ Another danger is the inadvertent de-prioritisation of TB care if the health system becomes inundated with people experiencing severe acute respiratory syndrome from infection with SARS-CoV-2. ${ }^{[12,13]}$ We run the risk of amplifying the effect when people with PTB return to communities without adequate treatment, with potential consequences well beyond the COVID-19 pandemic. It therefore behoves us as epidemiologists, clinicians and citizens to act now to stem the tide of COVID-19 transmission through our practice and communication. The World Health Organization recommends that TB patients follow all recommended precautions against COVID-19 and continue taking TB treatment throughout the pandemic. ${ }^{[12]}$ Ensuring continuity of $\mathrm{TB}$ care will require adequate stock of medication for patients to take home and the ramping up of digital technologies and other community support systems to provide outpatient and community care when it is safe to do so. ${ }^{[12]}$ Given that self-isolation may be a challenge to those at heightened risk, appropriate testing and the strict enforcement of infection control protocols, cough etiquette, and segregation of people who may have either TB or SARS-CoV-2 infection among in- and outpatients will be of paramount importance to limit further spread. TB-focused clinicians and health workers can also be vital points of reference for people infected with SARS-CoV-2 and for health staff undertaking active case finding and contact tracing. ${ }^{[12]}$

The spread of COVID-19 among people living with HIV is a wellfounded concern in SA. The implications of TB with and without HIV must equally be considered. Factors related to PTB and TB-HIV co-infection may complicate clinical suspicion for either COVID19 or PTB, and require clinical vigilance. Given the unknowns regarding COVID-19 in the context of SA's unique health burden, the resounding call to flatten the curve remains of critical importance.

Author contributions. JB researched and drafted the manuscript. TM and GS contributed to the concept of the manuscript and critically edited the content. SM, ZS, MP and AD critically edited the manuscript content.

\section{J Boffa}

McGill International TB Centre, McGill University, Montreal, Canada; and Centre for Rural Health, School of Nursing and Public Health, College of Health Sciences, University of KwaZulu-Natal, Durban, South Africa jody.boffa@mail.mcgill.ca

\section{T Mhlaba}

Department of Public Health Medicine, School of Nursing and Public Health, College of Health Sciences, University of KwaZulu-Natal, Durban, South Africa

\section{G Sulis}

McGill International TB Centre, McGill University, Montreal, Canada

\section{S Moyo}

Social Aspects of Public Health Research Programme, Human Sciences Research Council, Cape Town, South Africa; and School of Public Health, Faculty of Health Sciences, University of Cape Town, South Africa

\section{Z Sifumba}

St Andrews Hospital, Harding, KwaZulu-Natal, South Africa

\section{Pai}

McGill International TB Centre, McGill University, Montreal, Canada

\section{A Daftary}

Faculty of Health, York University, Toronto, Canada; and Centre for the AIDS Programme of Research in South Africa (CAPRISA), Durban, South Africa 
1. Preiser W, van Zyl G, Dramowski A. COVID-19: Getting ahead of the epidemic curve by early implementation of social distancing. S Afr Med J 2020;1 10(4):258. https://doi.org/10.7196/SAMJ.2020. v110i4.14720

2. Shisana O, Rehle T, Simbayi LC, et al. South African National HIV Prevalence, Incidence and Behaviou Survey, 2012. Cape Town: HSRC Press, 2014. http://ecommons.hsrc.ac.za/handle/20.500.11910/2490 Survey, 2012. Cape Town:
(accessed 25 March 2020)

3. National Department of Health, South Africa. Minister of Health Update [Twitter] 2020. https://twitter $\mathrm{com} /$ HealthZA/status/1242507959852302336? s=19 (accessed 24 March 2020).

4. National Department of Health, South Africa. Latest confirmed cases of COVID-19 (23rd March 2020). https://sacoronavirus.co.za/2020/03/23/latest-confirmed-cases-of-covid-19-23rd-march-2020/ (accessed 24 March 2020)

5. Huang C, Wang Y, Li X, et al. Clinical features of patients infected with 2019 novel coronavirus in Wuhan, China. Lancet 2020 (epub 24 January 2020). https://doi.org/10.1016/\$0140-6736(20)30183-5

6. Zhou F, Yu T, Du R, et al. Clinical course and risk factors for mortality of adult inpatients with COVID-19 in Wuhan, China: A retrospective cohort study. Lancet 2020 (epub 11 March 2020). https://do org/10.1016/S0140-6736(20)30566-3

7. World Health Organization. Global Health Observatory data repository: Antiretroviral coverage estimates by country: 2018. 2019. https://apps.who.int/gho/data/node.main.626 (accessed 25 March 2020).
8. World Health Organization. Country profiles: South Africa. In: Global Tuberculosis Report 2019. Geneva: WHO, 2019. https://www.who.int/tb/publications/global_report/en/ (accessed 24 March 2020). 9. Snider Jr DE. The relationship between tuberculosis and silicosis. Am Rev Respir Dis 1978;118:455-460. 1994:150(5):1460-1462. 1994;150(5):1460-1462.

11. Lawn SD, Bekker SG. Co-pathogenesis of tuberculosis and HIV. In: Schaaf HS, Zumla AI, eds. Tuberculosis: A Comprehensive Clinical Reference. London: Elsevier, 2009:96-106.

12. World Health Organization. WHO Information Note: Tuberculosis and COVID-19. 20 March 2020. https://www.who.int/tb/COVID_19considerations_tuberculosis_services.pdf (accessed 24 March 2020). 3. Pai M. COVID-19 coronavirus and tuberculosis: We need a damage control plan. Forbes, 17 March 2020. https://www.forbes.com/sites/madhukarpai/2020/03/17/covid-19-and-tuberculosis-we-need-a-damagecontrol-plan/\#70ea2496295c (accessed 24 March 2020).

S Afr Med J 2020;110(5):341-342. https://doi.org/10.7196/SAMJ.2020.v110i5.14747 